\title{
Unit of Arbitrary Substance Concentration
}

National Cancer Institute

\section{Source}

National Cancer Institute. Unit of Arbitrary Substance Concentration. NCI Thesaurus.

Code 667375.

A unit of measurement of arbitrary quantity of substance (based on its biologic activity) in the unit volume of the system. 